\title{
Near-infrared and optical observations of galactic warps: a common, unexplained feature of most discs
}

\author{
A. Guijarro ${ }^{1,2}$, R. F. Peletier ${ }^{3}$, E. Battaner ${ }^{1}$, J. Jiménez-Vicente ${ }^{1}$, R. de Grijs ${ }^{4,5}$, and E. Florido ${ }^{1}$ \\ 1 Dpto. Física Teórica y del Cosmos, Universidad de Granada, Granada, Spain \\ e-mail: aguijarr@ugr.es \\ 2 Centro Astronómico Hispano Alemán, Almería, Spain \\ Kapteyn Astronomical Institute, Groningen, The Netherlands \\ 4 Kavli Institute for Astronomy and Astrophysics and Department of Astronomy, Peking University, Beijing, PR China \\ 5 Department of Physics \& Astronomy, The University of Sheffield, UK
}

Received 26 March 2010 / Accepted 10 June 2010

\section{ABSTRACT}

\begin{abstract}
Context. Warps occurring in galactic discs have been studied extensively in HI and in the optical, but rarely in the near-infrared (NIR) bands that trace the older stellar populations.

Aims. We provide NIR data of nearby edge-on galaxies, combined with optical observations, for direct comparison of the properties of galactic warps as a function of wavelength, and calculate warp curves for each galaxy and obtain the characteristic warp parameters. We discuss these properties as possible constraints to the different mechanisms that have been proposed for the development and persistence of galactic warps.

Methods. We observed 20 galaxies that were selected from a statistically complete diameter-limited subsample of edge-on disc galaxies. We used the Cerro Tololo Infrared Imager (CIRIM) at the CTIO $1.5 \mathrm{~m}$ Ritchey-Chretien telescope to acquire the NIR data. We used the $1.54 \mathrm{~m}$ Danish and $0.92 \mathrm{~m}$ Dutch telescopes at the European Southern Observatory's La Silla site for our optical observations.

Results. Our results show that 13 of our 20 sample galaxies are warped, with the warp more pronounced in the optical than at NIR wavelengths. In the remaining seven galaxies, no warp is apparent within the limitations of our automated detection method. The transition between the unperturbed inner disc and the outer, warped region is rather abrupt. S0 galaxies exhibit very small or no warps. The magnetic model remains one of a number of interesting formation scenarios.
\end{abstract}

Key words. galaxies: general - galaxies: photometry - galaxies: structure

\section{Introduction}

Most spiral galaxies, including our own, exhibit warped discs. This has long been known based on observations of both the extended neutral-gas (HI) component (Sancisi 1976; Bosma 1981; Briggs 1990; García-Ruiz et al. 2002b) and optical starlight (Sánchez-Saavedra et al. 1990; Florido et al. 1991; Reshetnikov \& Combes 1998; Ann \& Park 2006). The first detections of warped discs came from $21 \mathrm{~cm}$ line observations of our own Galaxy (Burke 1957; Kerr 1957; Burton 1988). Based on a sky survey covering the Northern Hemisphere, Sánchez-Saavedra et al. (1990) first reported the high frequency of optical warps in disc galaxies, which they later confirmed in Sánchez-Saavedra et al. (2003) based on an extended catalogue that also included the Southern Hemisphere. These studies took into account that warps can only be detected when their orientation (projection) is favourable (i.e., not in the line of sight). A noteworthy and important result, established in the later paper, is that they did not find any warped S0 galaxies. Here, we therefore pay special attention to S0 galaxies. Reshetnikov et al. (2002) find that warps were also common in the past, with even greater magnitudes at $z \approx 1$.

Edge-on galaxies are usually chosen to study warps, although dynamical warps in the HI distribution of galaxies of intermediate inclination have also been reported, e.g., in M 83
(Rogstad et al. 1974), NGC 5033, NGC 5055, NGC 2841, and NGC 7331 (Bosma 1978), M 31 (Brinks \& Burton 1984), and M 33 (Corbelli et al. 1989). García-Ruiz et al. (2002b) analysed HI observations and find that all edge-on galaxies that have an extended HI disc (with respect to the optical component) are warped.

Ann \& Park (2006) find that $73 \%$ of the 325 galaxies in their well-defined sample of almost perfectly edge-on galaxies $(a / b>9.5$, where $a / b$ is the major-to-minor axis ratio at $\mu_{B}=25 \mathrm{mag}_{\operatorname{arcsec}}{ }^{-2}$ ) exhibit optical warps. Recently, Reylé et al. (2009) have found that a warp is present in the stellar, dust, and gas discs of the Milky Way, which are all asymmetric and characterised by a similar line of nodes. The Milky Way's HI gas warp is the strongest, followed by the dust warp, while the stellar warp is significantly smaller (by a factor of approximately two). They conclude that this comparison shows that the different components react differently to the forces responsible for the origin of the warp. The most important additional information that we provide here (not yet been discussed by other authors) is a multiwavelength study of galactic warps in one NIR and three optical filters.

Considerable effort has gone into establishing the physical origin and stability of warps. Numerous mechanisms have been proposed and explored, but they have thus far not provided a definitive and satisfactory physical explanation (see, e.g., the 
reviews by Binney 1992; Battaner et al. 1997; van der Kruit 2007). Kerr (1957), Hunter \& Toomre (1969), Weinberg (1998), Weinberg \& Blitz (2006), and others have suggested that warps can result from tidal interactions with a satellite or neighbouring galaxy. This hypothesis can explain warps neither in isolated galaxies (Sancisi 1976; Tubbs \& Sanders 1979; Sparke 1984) nor in the Milky Way, if the latter was produced by an interaction with the Large Magellanic Cloud (see García-Ruiz et al. 2002a). Hunter \& Toomre (1969) and Dekel \& Shlosman (1983) proposed that the Galactic warp is a result of oscillations in the disc, either triggered by an interaction with another galaxy in the past or caused by the disc being embedded in a nonspherical halo of dark matter. Sparke \& Casertano (1988) showed that some discrete oscillation modes can survive. This theory is not without problems, however; for instance, Binney (1991) showed that the halo should then respond to its misalignment with the disc, thus destroying the warp in a few orbital periods. Alternatively, a nonlinear coupling between a spiral wave and two warp waves could create a warp (Masset \& Tagger 1997). The efficiency of this mechanism is too low in the stellar disc except at its outer edge (near a Lindblad resonance), where the spiral wave slows down and is coupled efficiently to the warp waves. At those radii, the energy of the spiral arm can almost be completely converted into transmitted and reflected warp waves, which can be observed as corrugations in the disc.

Warping could thus be a natural response of the outer disc to a series of stimulations, and it seems that the responsible mechanism does not necessarily have to be the same in each galaxy. In any case, observations of large edge-on galaxies could contribute to the definitive determination of the dominant scenario. The intergalactic medium could also play an important role by accretion of matter as galaxies move along their paths (Kahn \& Woltjer 1959; López-Corredoira et al. 2002; Sánchez-Salcedo 2006). Battaner et al. (1990) explained warps by intergalactic magnetic fields, which would produce a direct distortion in the distribution of the gas. The stellar system would also indirectly reach a warped distribution because stars are formed from gas, but this would result in a difference between young and old stars. Colour gradients in warps can thus provide an important clue. Based on this model, gas-poor S0 galaxies should not exhibit warps.

In this paper, we present new, deep NIR and optical observations of 20 edge-on galaxies. NIR wavelengths have rarely been used to date to study warps. They are, however, essential for better tracing the stellar mass distribution, because at infrared (IR) wavelengths $(1<\lambda<5 \mu \mathrm{m})$ dust extinction is minimised (the absorption, in magnitudes, in the $K$ filter is $10 \%$ of that in the $V$ band, $\left.A_{K} / A_{V} \sim 0.1\right)$. NIR emission is also a better tracer of old stellar populations, one of our major objectives in this study. Therefore, these wavelengths provide essential complementary information for studying stellar warps.

\section{Sample}

Our sample was selected by de Grijs $(1997,1998)$ from the Surface Photometry Catalogue of the ESO-Uppsala Galaxies (ESO-LV; Lauberts \& Valentijn 1989). This catalogue contains large numbers of galaxies that have been selected and parameterised uniformly. The galaxies were originally selected on ESO-Schmidt survey plates covering declinations $\delta \leq-17.5^{\circ}$, excluding the area within $15^{\circ}$ of the Galactic Equator, at Galactic latitudes $|b|<15^{\circ}$. We selected 20 candidate galaxies from a statistically complete diameter-limited subsample of edge-on disc galaxies (de Grijs 1997, 1998), with the following characteristics (see Table 1):

- $D_{25}(B) \geq 2.2^{\prime}$, i.e., with optical diameters (at a surface brightness level of $\mu_{B}=25 \mathrm{mag} \operatorname{arcsec}^{-2}$ ) larger than 2.2';

- isolated galaxies, i.e., without significant neighbours within a distance greater than or equal to five times their optical diameters. They should be classified as noninteracting and undisturbed;

- inclination, $i>87^{\circ}$;

- morphological type range from S0 to Sd, i.e., revised Hubble types later than $T=-2$ (spiral and lenticular galaxies).

The inclinations were determined assuming an intrinsic flattening $(b / a)_{0}=0.11$ (de Grijs 1997, 1998), so that our sample contains highly inclined galaxies in which warps should be easy to detect. This could introduce a bias against early types or galaxies with prominent bulges that should be taken into account in the discussion of the warp phenomena. Using this approach, we try to avoid the common confusion between spiral arms, corrugations, and warps.

\section{Observations and data reduction}

The sample was observed at

- near-infrared wavelengths, with the Cerro Tololo Infrared IMager (CIRIM) at the $1.5 \mathrm{~m}$ Ritchey-Chretien Telescope of the Cerro Tololo Inter-American Observatory (CTIO) on October 22-27, 1998 (Prop0020). CIRIM is equipped with a $256 \times 256$-pixel HgCdTe NICMOS3 array (Rockwell Science) with a pixel scale of $1.16^{\prime \prime}$. We observed our sample galaxies through the $K_{\text {short }}$ filter $\left(\equiv K_{\mathrm{S}}\right)$, characterised by an effective wavelength $\lambda_{\text {eff }}=2.15 \mu \mathrm{m}$ (Wainscoat \& Cowie 1992). We opted for this filter instead of the longer-wavelength $K$ filter $\left(\lambda_{\text {eff }}=2.20 \mu \mathrm{m}\right)$ because the sky brightness in $K_{\mathrm{s}}$ is lower than in the $K$ band (for instance, at UKIRT, Hawaii, the sky brightness is 13.5 and $13.0 \mathrm{mag} \operatorname{arcsec}^{-2}$ in $K_{\mathrm{s}}$ and $K$, respectively ${ }^{1}$ ) and $K_{\text {short }}$ is almost as little affected by dust as the $K$ band, $A_{K_{\mathrm{s}}} \sim$ $0.11 \mathrm{mag}^{\text {airmass }}{ }^{-1}$; and

- optical wavelengths. We obtained our observations using two telescopes of the European Southern Observatory (ESO) at La Silla, Chile, including the $1.54 \mathrm{~m}$ Danish telescope (equipped with a $1081 \times 1040$-pixel TEK CCD with $0.36^{\prime \prime}$ pixel $^{-1}$ ) and the $0.92 \mathrm{~m}$ Dutch telescope (equipped with a $512 \times 512$-pixel TEK CCD with $0.44^{\prime \prime}$ pixel $^{-1}$ ), both in the standard Johnson $B$ and $V$ and Thuan \& Gunn (1976) $i$ filters.

The observations and optical data-reduction techniques on which the results presented in this paper are based were described in detail in de Grijs (1998). Here, we focus on the reduction of the IR data.

To study stellar warps in disc galaxies, we need to obtain reliable data at the very low surface brightnesses in their outermost regions. Our observational data reached surface brightnesses fainter than $26 \mathrm{mag} \operatorname{arcsec}^{-2}$ in the $B$ band. This is only $6 \%$ of the sky brightness $\left(\mu_{B, \text { sky }}=23 \mathrm{mag} \mathrm{arcsec}^{-2}\right)$, so that we had to be particularly careful in measuring the sky brightness. The sky background in NIR passbands is much higher than in the optical $\left(\mu_{K, \text { sky }}=13 \mathrm{mag} \operatorname{arcsec}^{-2}\right)$ and can change significantly

\footnotetext{
1 http://www . jach.hawaii . edu/UKIRT/instruments/uist/ imaging/imaging.html
} 
A. Guijarro et al.: Near-infrared and optical observations of galactic warps

Table 1. Global galaxy parameters from the ESO-LV Catalogue (Lauberts \& Valentijn 1989).

\begin{tabular}{|c|c|c|c|c|c|c|c|c|c|}
\hline Galaxy & $\begin{array}{l}\text { RA(J2000.0) } \\
\text { (hh:mm:ss.s) }\end{array}$ & $\begin{array}{c}\operatorname{Dec}(\mathrm{J} 2000.0) \\
\text { (dd:mm:ss) }\end{array}$ & $\begin{array}{r}\text { Hubble } \\
\text { type }\end{array}$ & $\begin{array}{c}a / b \\
\text { (axis ratio) }\end{array}$ & $\begin{array}{r}D_{25}(B) \\
(\operatorname{arcsec})\end{array}$ & $\begin{array}{l}B \text { mag } \\
\text { (total) }\end{array}$ & $\begin{array}{c}B-R \text { (tot) } \\
(\mathrm{mag})\end{array}$ & $\begin{array}{l}\text { Radial velocity } \\
\left(\mathrm{km} \mathrm{s}^{-1}\right)\end{array}$ & $\begin{array}{r}\text { PA (deg) } \\
(\mathrm{N} \rightarrow \mathrm{E})\end{array}$ \\
\hline ESO026-G06 & $20: 48: 28.3$ & $\begin{array}{l}-78: 04: 09 \\
\end{array}$ & $6.7 \pm 1$ & 9.3 & 134.9 & $15.48 \pm 0.30$ & +1.37 & $2323^{1)}$ & 76.0 \\
\hline ESO033-G22 & $05: 31: 41.8$ & $-73: 44: 58$ & $6.8 \pm 1$ & 12.5 & 131.8 & $15.56 \pm 0.19$ & +0.86 & $3932^{1)}$ & 170.0 \\
\hline ESO142-G24 & $19: 35: 42.3$ & $-57: 31: 10$ & $6.6 \pm 1$ & 8.4 & 237.1 & $14.03 \pm 0.24$ & +1.30 & $2027^{1)}$ & 6.0 \\
\hline ESO157-G18 & $04: 17: 54.4$ & $-55: 56: 04$ & $6.5 \pm 1$ & 6.6 & 186.2 & $13.90 \pm 0.48$ & +0.96 & 1139 & 18.0 \\
\hline ESO201-G22 & 04:09:00.4 & $-48: 43: 36$ & $4.8 \pm 1$ & 7.3 & 151.4 & $14.73 \pm 0.32$ & $\cdots$ & 4014 & 59.0 \\
\hline ESO202-G35 & $04: 32: 15.6$ & $-49: 40: 31$ & $3.4 \pm 1$ & 6.2 & 184.1 & $13.38 \pm 0.31$ & +1.06 & 1856 & 133.0 \\
\hline ESO235-G53 & $21: 05: 10.4$ & $-47: 47: 17$ & $3.0 \pm 0$ & 6.0 & 153.1 & $14.53 \pm 0.26$ & +1.85 & 5110 & 49.0 \\
\hline ESO240-G11 & $23: 37: 49.3$ & $-47: 43: 34$ & $4.7 \pm 1$ & 10.0 & 331.1 & $13.05 \pm 0.21$ & +1.35 & 2817 & 129.0 \\
\hline ESO288-G25 & $21: 59: 17.6$ & $-43: 52: 02$ & $4.2 \pm 1$ & 6.5 & 153.1 & $13.97 \pm 0.21$ & +1.30 & 2484 & 53.0 \\
\hline ESO311-G12 & $07: 47: 34.2$ & $-41: 27: 07$ & $0.1 \pm 1$ & 6.4 & 251.2 & $12.83 \pm 0.28$ & +1.69 & $848^{1)}$ & 14.0 \\
\hline ESO340-G08 & $20: 17: 11.0$ & $-40: 55: 22$ & $6.0 \pm 1$ & 24.0 & 192.8 & $15.45 \pm 0.20$ & +0.95 & $3004^{1)}$ & 34.0 \\
\hline ESO340-G09 & $20: 17: 20.2$ & $-38: 40: 29$ & $7.0 \pm 1$ & 9.0 & 160.3 & $14.52 \pm 0.27$ & $\ldots$ & 2546 & 98.0 \\
\hline ESO358-G26 & 03:35:30.8 & $-34: 26: 49$ & $-1.6 \pm 2$ & 4.2 & 153.1 & $12.90 \pm 0.40$ & +1.19 & 1660 & 84.0 \\
\hline ESO358-G29 & $03: 36: 31.4$ & $-35: 17: 38$ & $-2.0 \pm 1$ & 3.9 & 153.1 & $12.47 \pm 0.18$ & +1.45 & 1740 & 139.2 \\
\hline ESO416-G25 & $02: 48: 41.3$ & $-31: 32: 09$ & $3.2 \pm 1$ & 6.0 & 141.3 & $14.68 \pm 0.25$ & +1.38 & 4992 & 31.0 \\
\hline ESO460-G31 & $19: 44: 21.2$ & $-27: 24: 22$ & $4.8 \pm 1$ & 9.5 & 154.9 & $15.15 \pm 0.21$ & $\ldots$ & $5352^{1)}$ & 92.0 \\
\hline ESO487-G02 & $05: 21: 48.6$ & $-23: 48: 45$ & $3.9 \pm 1$ & 7.5 & 199.5 & $13.62 \pm 0.26$ & +1.35 & $2088^{1)}$ & 60.0 \\
\hline ESO531-G22 & $21: 40: 29.5$ & $-26: 31: 40$ & $4.5 \pm 1$ & 8.7 & 154.2 & $13.94 \pm 0.35$ & $\cdots$ & $3639^{1)}$ & 8.0 \\
\hline ESO555-G36 & $06: 07: 41.5$ & $-19: 54: 54$ & $5.5 \pm 1$ & 11.5 & 139.6 & $15.60 \pm 0.58$ & +1.62 & $\ldots$ & 146.0 \\
\hline ESO564-G27 & 09:11:54.3 & $-20: 07: 04$ & $6.1 \pm 1$ & 14.0 & 278.6 & $14.39 \pm 0.90$ & +1.36 & 2178 & 168.0 \\
\hline
\end{tabular}

Notes. ${ }^{1)}$ Heliocentric radial velocities from Mathewson et al. (1992).

on temporal and spatial scales the order of two minutes and two arcmin, respectively. It was therefore necessary to obtain as many sky frames as object frames to perform sky subtraction with sufficiently high accuracy. We took sky and object frames alternately, using the same exposure time for each individual frame $(60 \mathrm{~s})$ and employing interframe shifts of $\sim 2 \operatorname{arcmin}$. An additional reason we needed to obtain separate sky frames is that the IR arrays used were not large enough to properly sample the sky on the science frames. Incorrect background subtraction can cause the sky level to be either underestimated (so that sky flux will contribute to the surface brightness at large galactic radii) or overestimated (resulting in a fictitious radial cut-off). Figure 1 illustrates the importance of careful background subtraction in the $K_{\mathrm{s}}$ band, where the sky contribution is greater than in optical passbands. The figure shows the major- and the minor-axis (vertical, $z$ ) surface brightness profiles of four galaxies, binned radially. The effects of oversubtraction can be seen clearly in the minor-axis surface brightness profiles: they show artificial cut-offs, and the negative background values result in undefined surface brightnesses at these $z$ heights.

We used a special software package for our NIR data reduction, customised to reduce and analyse very deep data of extended objects observed in the IR, as a set of IRAF ${ }^{2}$ tasks. The program removes spurious stars in sky frames, subtracts cleaned, scaled sky frames from object frames taken immediately prior to or after the relevant object frame, and flatfields the differences. It uses a median sky frame to detect and remove stars in the sky frames.

We observed several standard stars from the SAAO/ESO/ISO Faint Standard Stars Catalogue (Carter \& Meadows 1995) to calibrate our targets and used the Wainscoat \& Cowie (1992) correction to convert $K_{\mathrm{s}}$ to $K$ magnitudes. These stars were observed at least three times per night, at different airmasses, to calculate the prevailing atmospheric

\footnotetext{
${ }^{2}$ IRAF is distributed by the National Optical Astronomy Observatories, which are operated by the Association of Universities for Research in Astronomy, Inc., under cooperative agreement with the US National Science Foundation.
}

extinction with sufficient accuracy. We observed a total of 21 standard stars. These observations reached a surface brightness of approximately $23 \mathrm{mag} \operatorname{arcsec}^{-2}$ in $K_{\mathrm{s}}$.

\section{Data analysis}

First, we aligned the images in all of the $B, V, I$, and $K_{\mathrm{s}}$ filters. We took special care to subtract foreground stars. This is necessary and important because foreground stars projected close to a galaxy can contaminate the galaxy's luminosity distribution and lead to erroneous results. We used a master mask constructed by adding the individual masks for each of the passbands. In each mask, the stars were marked with circles of radius around twice the FWHM to avoid contributions from any residual starlight. In some galaxies observed near the Galactic plane, this meant that a large number of pixels had to be masked.

To determine the warp curve (tracing the position of the intensity maxima as a function of radius), we employed three different methods.

1. First, we calculated the intensity maxima using vertical cuts (perpendicular to the galactic plane). This resulted in very noisy profiles for the optical data and also sometimes for the NIR data, because the presence of a dust lane frequently resulted in a double-peaked luminosity distribution.

2. Second, we calculated the luminosity distribution's first moment, with which we obtained reliable results (although it also depended on the position of the dust lane). This method was applied mainly at blue wavelengths to compensate for background stars.

3. Third, we fitted Gaussian profiles. We fitted each vertical trace (parallel to the minor axis of the galaxy) in the selected zone to a Gaussian function. We considered this the best approach, because the contribution of the dust lane is less important in optical data than when using the other two methods. Gaussians can be matched to vertical profiles that contain a dip caused by a dust lane without problems in almost perfectly edge-on galaxies. 

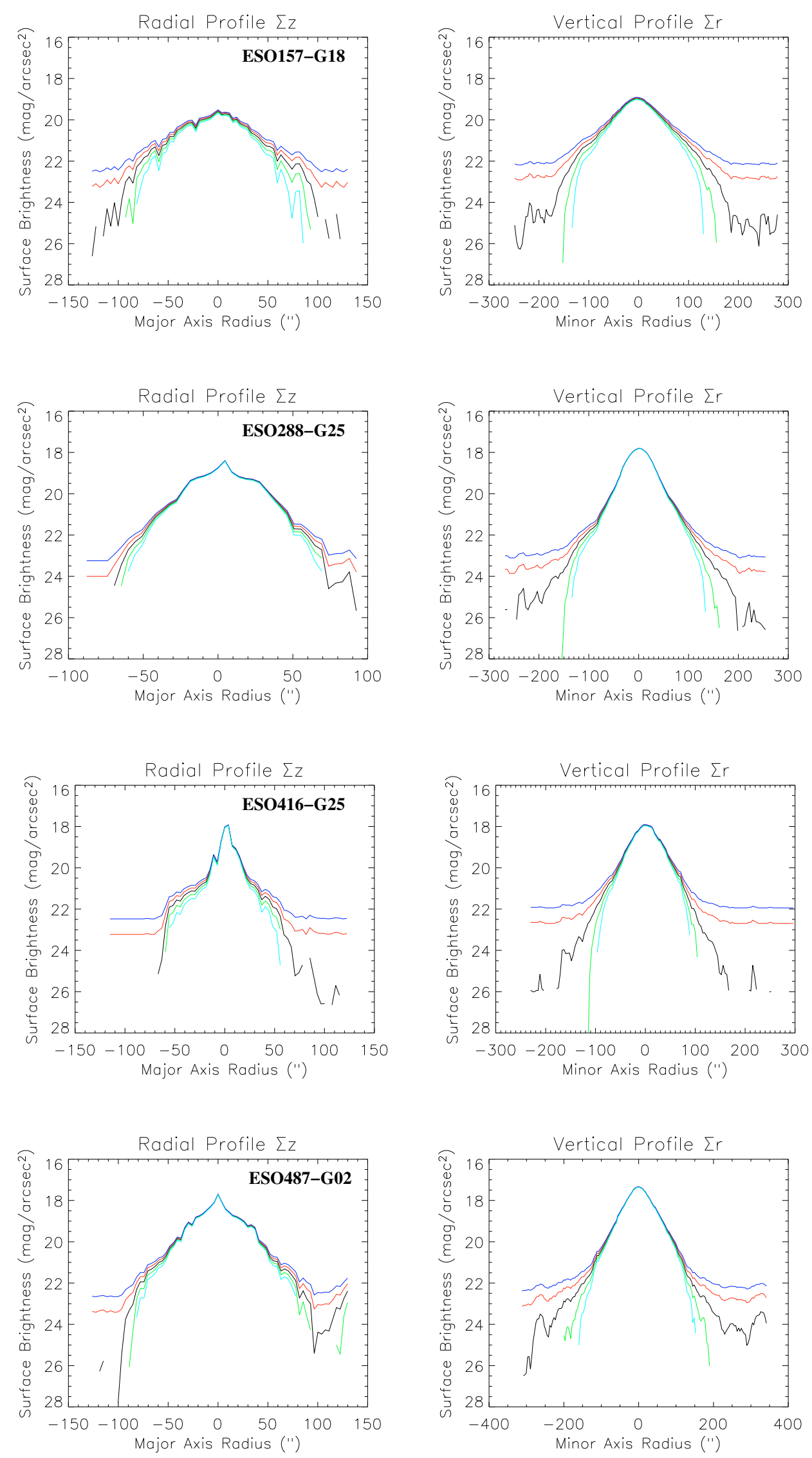

Fig. 1. $K_{\mathrm{s}}$-band luminosity profiles of four galaxies. The lines in each panel represent, from top to bottom, $-2 \sigma$ (blue), $-1 \sigma$ (red), profile after subtraction of our best estimate of the sky background (black), $+1 \sigma$ (green), and $+2 \sigma$ (sky blue). ( $\sigma$ is the sky noise in the regions we used to determine the sky-background levels.) 


\subsection{Warp curves from the Gaussian fit}

As a first step, we rotated our galaxies (to align their major axes with the horizontal axis) using an iterative method until the position angle (PA) had been determined to an accuracy of approximately 0.05 degrees. We used the PA from the ESO-LV Catalogue as a starting point; we then iteratively fitted a straight line to the central region of the galaxy. Our results are shown in Fig. 2 as isophote maps of our 20 galaxies.

To calculate the warp curves, we fitted Gaussians to the vertical profiles of the galaxies in each filter. We only considered data with a signal-to-noise ratio greater than $3\left(\sigma=\sqrt{\sigma_{\text {sys }}^{2}+\sigma_{\text {std }}^{2}}\right.$, where $\sigma_{\text {sys }}$ is the systematic error associated with the random background noise and $\sigma_{\text {std }}$ the standard deviation), and with the FWHM of the peak smaller than 100 pixels. The results of our analysis are presented in Fig. 3, where the warp curves are shown only for those values with error bars smaller than $0.5^{\prime \prime}$. This estimated error bar can be computed by scaling the standard deviation ( $1 \sigma$ error) by the measured $\chi^{2}$ value.

\subsection{Warp parameters}

It is convenient to use a geometric definition of a warp, e.g., for statistical studies. For an edge-on galaxy, the suitable coordinates correspond to the two directions contained in the plane of the sky: the direction defined by the major axis of the galaxy, $x$, and the direction of the rotation axis of the galaxy, $y$. The centre of the galaxy is the origin of coordinates. A warp curve is defined as the locus of points $\left(x_{i}, y_{i}\right)$ tracing the distribution of the highest intensities, where $y_{i}$ is the position of the centre of the best-fitting Gaussian at a given $x_{i}$.

We derived the parameter $\omega$ (defined in Jiménez-Vicente et al. 1997),

$\omega=\frac{1}{L^{3}} \int_{-L / 2}^{+L / 2} x y \mathrm{~d} x$,

where $L$ is the full size of the edge-on galaxy (the diameter at $3 \sigma$ above the sky background). The absolute value of $\omega$ is a measurement of the strength of a warp, while its sign distinguishes between N-like $(\omega>0)$ and S-like $(\omega<0)$ warps. These types are defined from the similarity of the warp shape and the type letters (see Fig. 4 for definition of types of warps). This definition is nondimensional and, therefore, independent of a galaxy's distance and size. It is also independent of the units used and does not strongly depend on the angular resolution of the observations (although a higher resolution would enable a more precise evaluation). For numerical purposes, we have

$\omega=\frac{1}{L^{3}} \sum_{i=-L / 2}^{L / 2} x_{i} y_{i}$.

We can also define this parameter for both sides of our galaxies: $\omega_{\mathrm{R}}$ for the right-hand side $\left({ }^{1} / L^{3} \sum_{i=0}^{L / 2} x_{i} y_{i}\right)$ and $\omega_{\mathrm{L}}$ for the left-hand side $\left(1 / L^{3} \sum_{i=-L / 2}^{0} x_{i} y_{i}\right)$. Because of the flattening of the radial profile in the inner regions of our sample galaxies, it is not critical to have a precise estimate of the position of the galaxy centre. Warps can appear in three flavours (cf. López-Corredoira et al. 2008). The N- or S-like warps have $\omega_{\mathrm{R}}$ and $\omega_{\mathrm{L}}$ of the same sign, the signs of $\omega_{R}$ and $\omega_{L}$ for U-like warps differ, and L-like warps apply to galaxies in which only one side is warped. The difference between $\mathrm{N}$ - and $\mathrm{S}$-like warps is unimportant from a physical point of view. However, we have retained this information because it is needed, for instance, when considering the orientation of warps in a cluster of galaxies (see, e.g., Battaner et al. 1991).

With this parameter we can, for example, objectively compare the appearance of a galaxy at several wavelengths to determine whether a colour gradient exists within the warp. We can also detect warps that would otherwise not have been found. Warps are not always completely symmetric, in which case this parameter would hide the information of the clearly warped side of the galaxy. To avoid this problem, we calculated the warp parameters on either side of our galaxies independently, along with $\alpha_{\mathrm{s}}$, the degree of asymmetry (Sánchez-Saavedra et al. 2003),

$\alpha_{\mathrm{s}}=\frac{\left|\omega_{\mathrm{R}}-\omega_{\mathrm{L}}\right|}{\omega_{\mathrm{R}}+\omega_{\mathrm{L}}}$

One of the most important errors in estimating $\omega$ arises from imperfectly determining the PA of the major axis, resulting in an error $\theta$ in the PA. Since $\theta \neq 0$, we introduce the error

$\Delta \omega=\frac{1}{L^{3}} \int_{-\infty}^{\infty} x(x \tan \theta) \mathrm{d} x \approx \frac{1}{3 L^{3}} L^{3} \theta \approx \frac{\theta}{3}$.

As $\theta$ is, in general, approximately $0.05^{\circ}\left(9 \times 10^{-4}\right.$ radians $)$, we estimate $\Delta \omega$ to be of the order of $3 \times 10^{-4}$ rad. A large warp can have a value of $10 \times 10^{-4}$ and a barely perceptible warp $5 \times 10^{-4} \mathrm{rad}$. In the remainder of this paper, we consider a galaxy as warped if its calculated $\omega$ is clearly greater than the associated error. (See Table 2 for our results.)

For those galaxies showing an appreciably warped disc, we fitted a number of additional parameters to the warp curves. We tried to use the function proposed by Jiménez-Vicente et al. (1997), but it had too many fitting parameters so the fits were resticted to the simple description:

$y= \begin{cases}0 & |x|<|A| \\ C(x-A) & |x| \geq|A|\end{cases}$

This function reproduces the warp's shape as a first approximation; i.e., it is flat up to a point and then deviates from the symmetry plane until it asymptotically reaches a new direction. The interpretation of the parameters $A$ and $C$ is as follows,

- $A$ is the starting point of the warp. It has dimensions of length;

- $C$ is the (nondimensional) value of the asymptotic slope.

Throughout the rest of the paper, we refer to parameters A and $\mathrm{C}$ as those obtained by fitting the experimental points to Eq. (5). These values are shown in Table 3 for the different bands. In addition, we use the angles $\alpha$ and $\beta$, which are most widely used to represent the warp amplitude (Sánchez-Saavedra et al. 2003) Here, $\alpha$ is the angle between the line connecting the galaxy's centre and the outermost detected point, and the disc's major axis, while $\beta$ is the angle between the disc's major axis and the line from the outermost detected point to the point where the warp starts $(A)$ : see Fig. 4.

We obtained the same result for the warp parameter $\omega$, whether it was calculated starting from the galaxy centre or from the starting point of the warp, $A$. This confirms that it is not critical to have a precise estimate of the position of the galaxy centre because of the radial flattening in the inner regions of our galaxies. In some galaxies, such as ESO142-G24 or ESO157-G18, the warp sets off in one direction, turns back to the mean plane, and ends in the opposite hemisphere. The Milky Way's warp 
A\&A 519, A53 (2010)


Fig. 2. Contour maps of our 20 galaxies in the four filters analysed. The isophotes are equidistant (in units of $n \times 3 \sigma$ with $n=1,2,3$, .., equivalent to steps of $0.75 \mathrm{mag} \operatorname{arcsec}{ }^{-2}$ ), starting at $\sim 3 \sigma$ above the sky background. The galaxies have been rotated, but $\mathrm{N}$ is close to the top, $\mathrm{E}$ to the left. 
A. Guijarro et al.: Near-infrared and optical observations of galactic warps
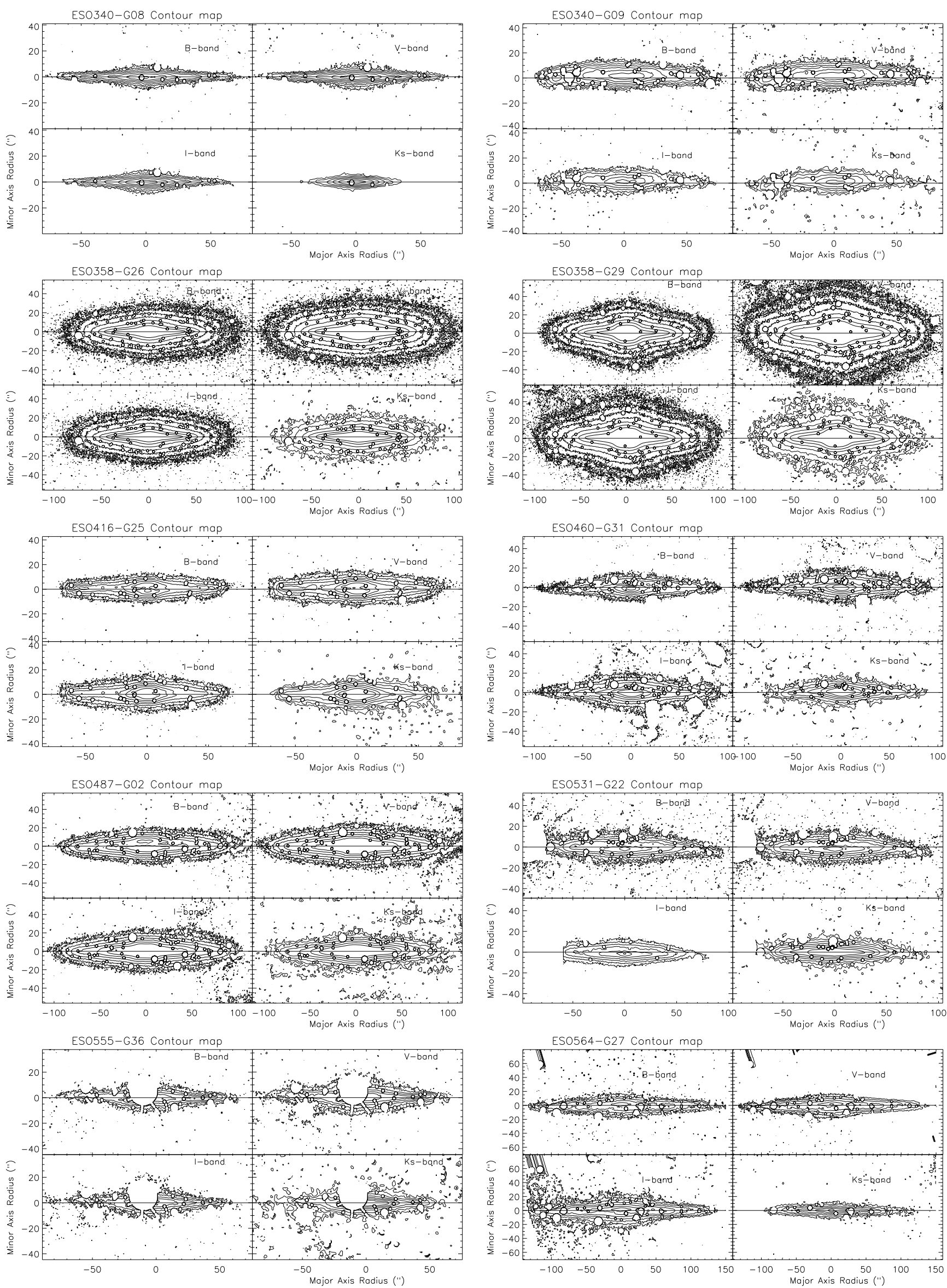

Fig. 2. continued. 
A\&A 519, A53 (2010)
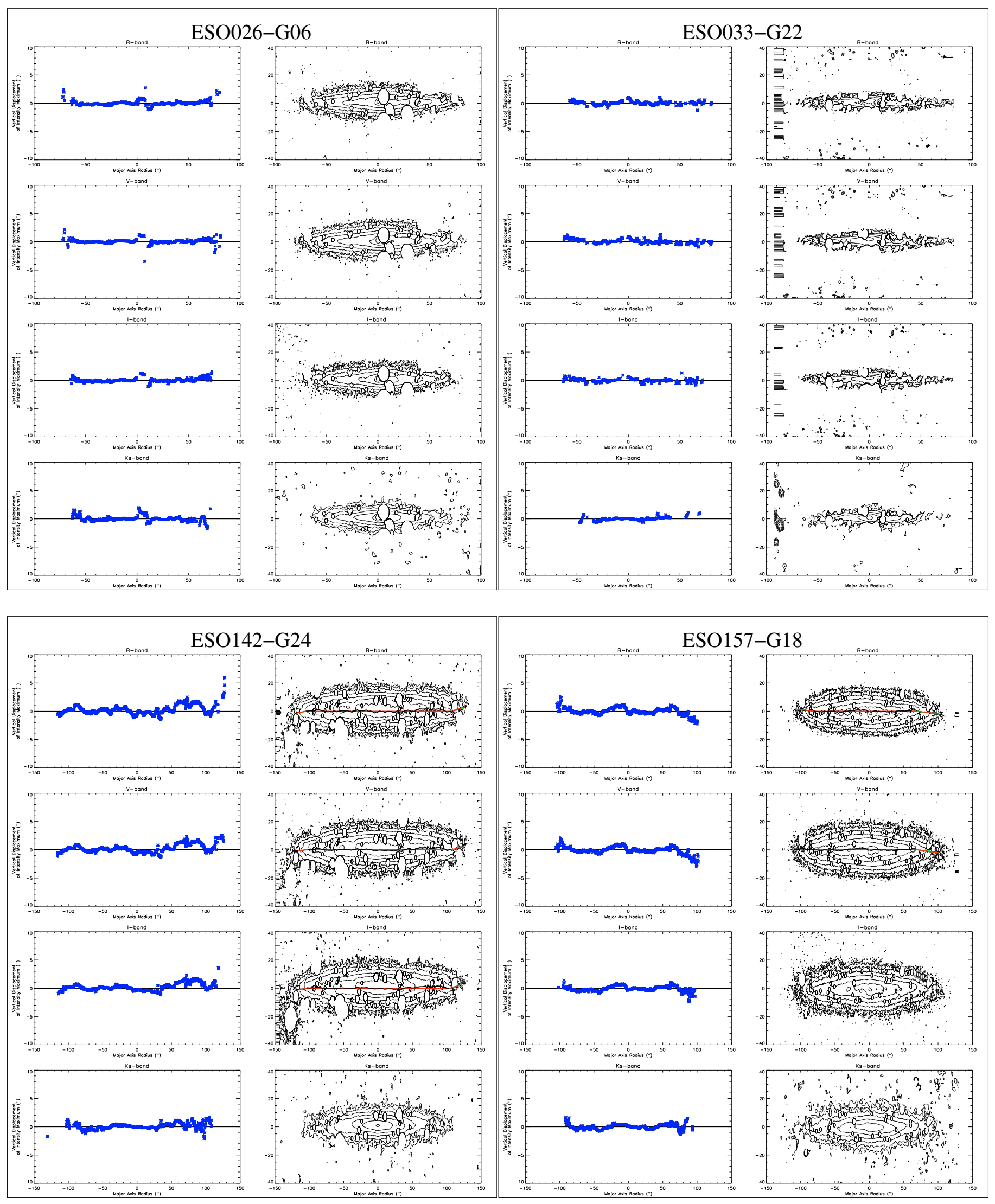

Fig. 3. Left: warp curves and right: contour maps, for the different filters of our 20 galaxies. The warp curve (green line) is put on top only for those values with an error bar less than $0.5^{\prime \prime}$. The red line shows the fitted warp curve. The isophotes are equidistant (in units of $n * 3 \sigma$ equiv. to a step of $+0.75 \mathrm{mag} / \operatorname{arcsec}^{2}$ ) starting at a level of about $3 \sigma$ above the sky background. The galaxy is rotated, but $\mathrm{N}$ is closer to the top and $\mathrm{E}$ to the left. The scale on the $y$-axis is enlarged to show a better curve. 
A. Guijarro et al.: Near-infrared and optical observations of galactic warps

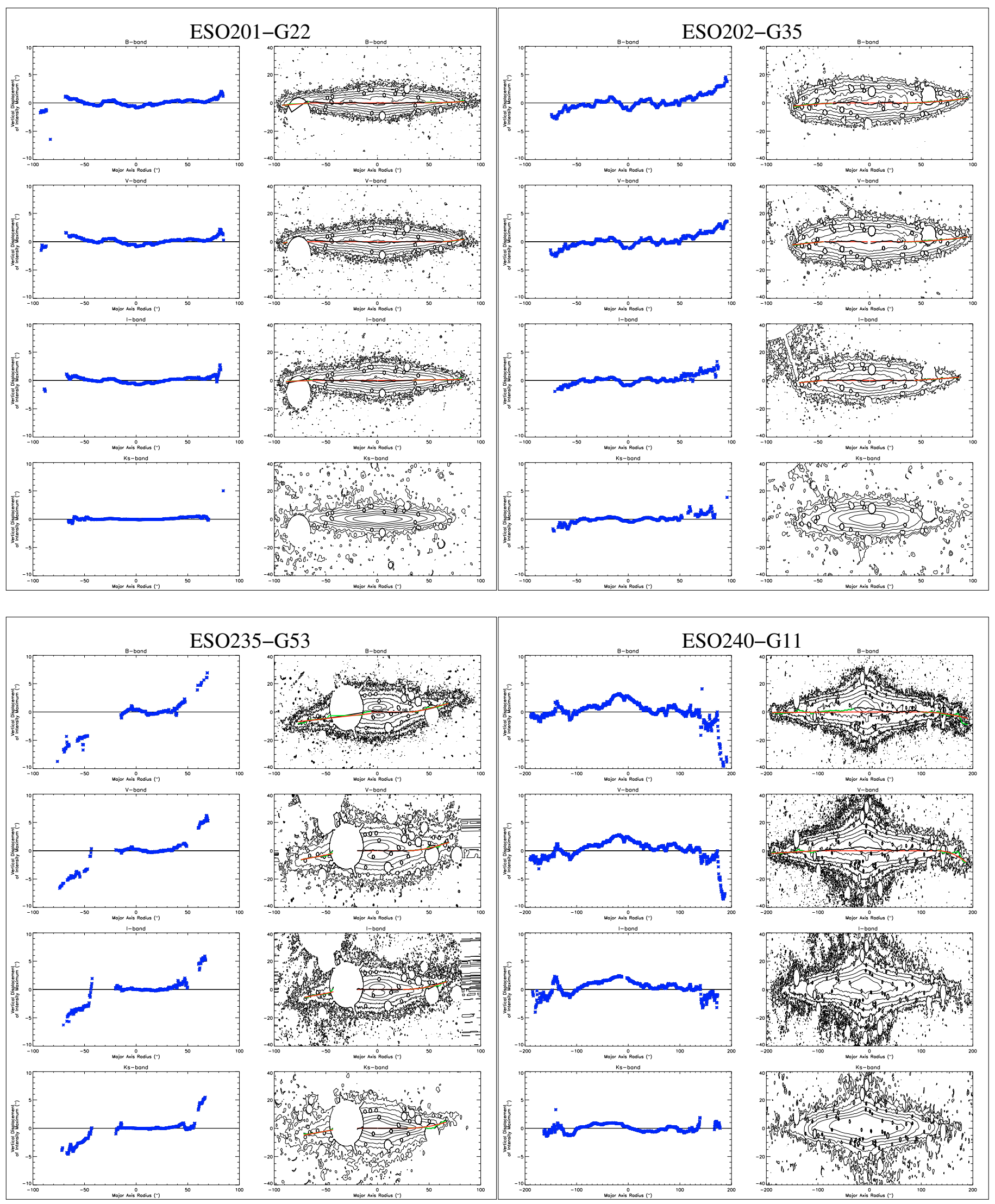

Fig. 3. continued.

in the Southern Hemisphere is an example of this type of behaviour (Porcel et al. 1997). Florido et al. (1991) also report such "elbow"-type warps. This effect may come from the presence of a more strongly warped dust lane (than the stellar disc) or be an intrinsic effect associated with warps. For such galaxies, a fourparameter fit would have provided a better description. However, 

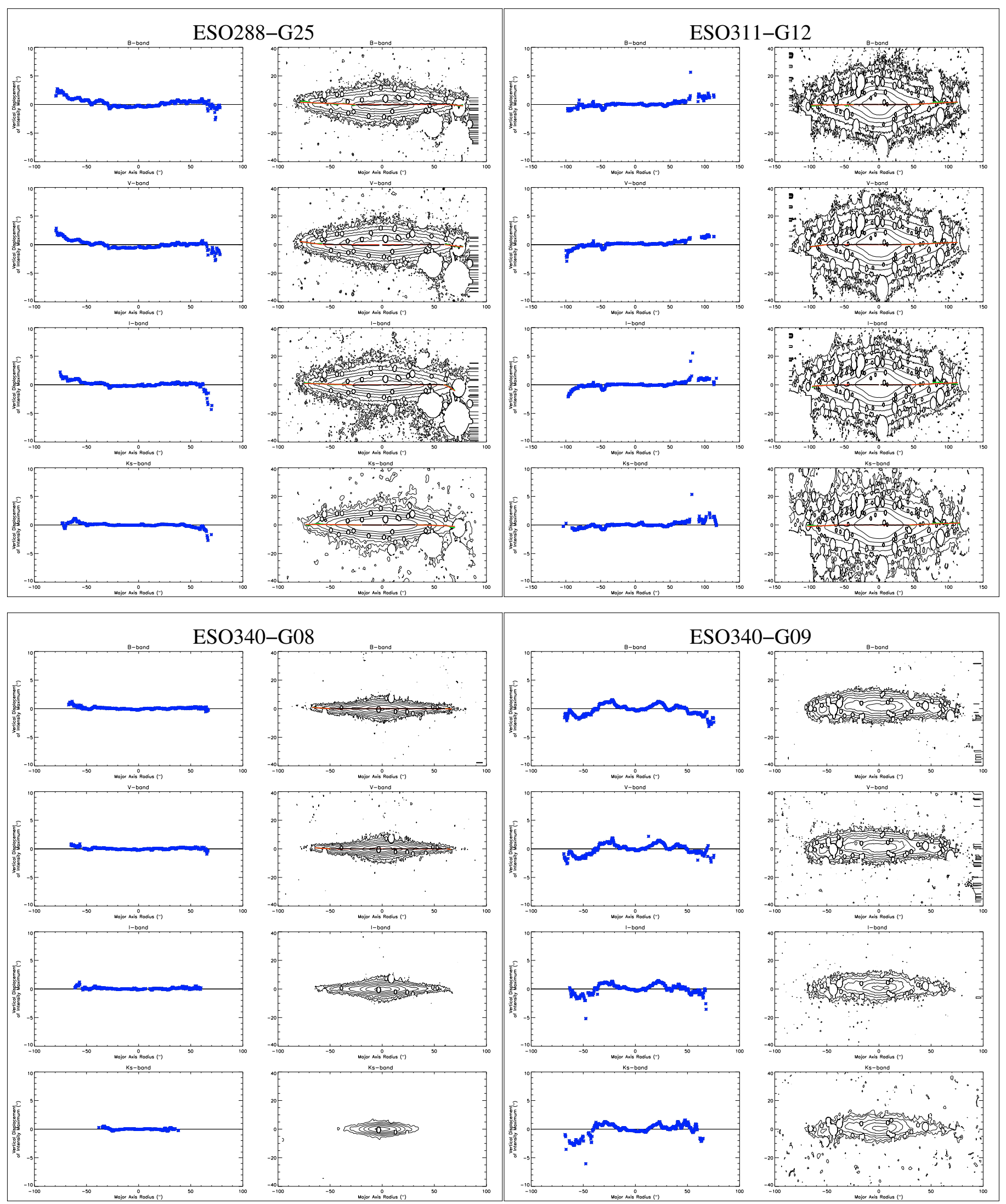

Fig. 3. continued.

since an excessive number of fit parameters renders any interpretation of simple warps unclear, we prefer to use three-parameter fits.

Page 10 of 16

\section{Analysis}

We consider a galaxy as warped if it is warped (according to the definition in Sect. 4.2) in any of the four observed bands. Based 
A. Guijarro et al.: Near-infrared and optical observations of galactic warps
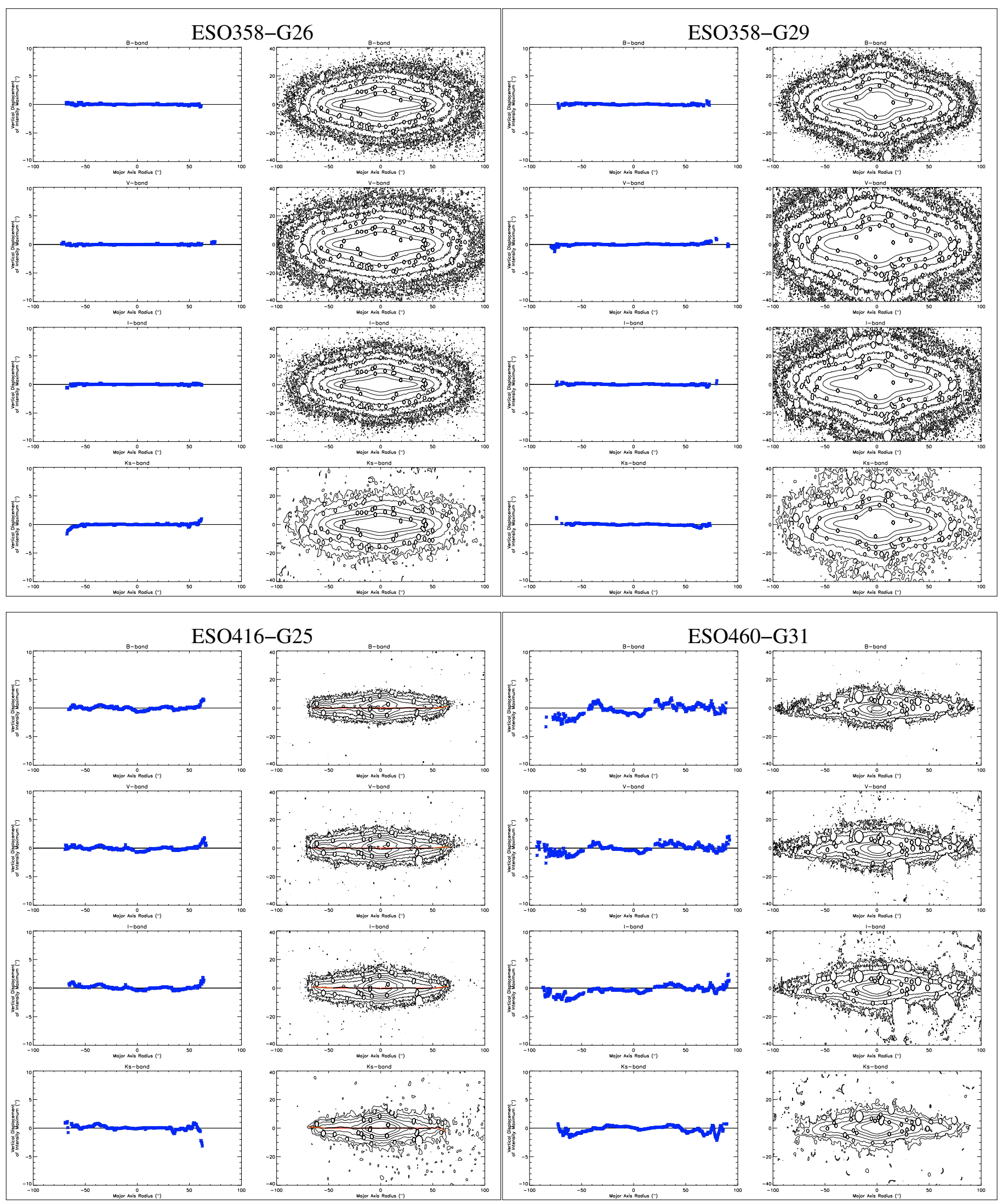

Fig. 3. continued.

on this assumption, we find that 13 of our 20 sample galaxies $(65 \%)$ are warped. This fraction supports previous results based on larger galaxy samples with similar optical characteristics to our sample (Sánchez-Saavedra et al. 1990, 2003; Reshetnikov \&
Combes 1999). Given the difficulties of observing warps with their line of nodes perpendicular to the line of sight, this high fraction of warped discs indicates that essentially all spirals are warped. Optical warps are less perceptible than HI warps, but at 

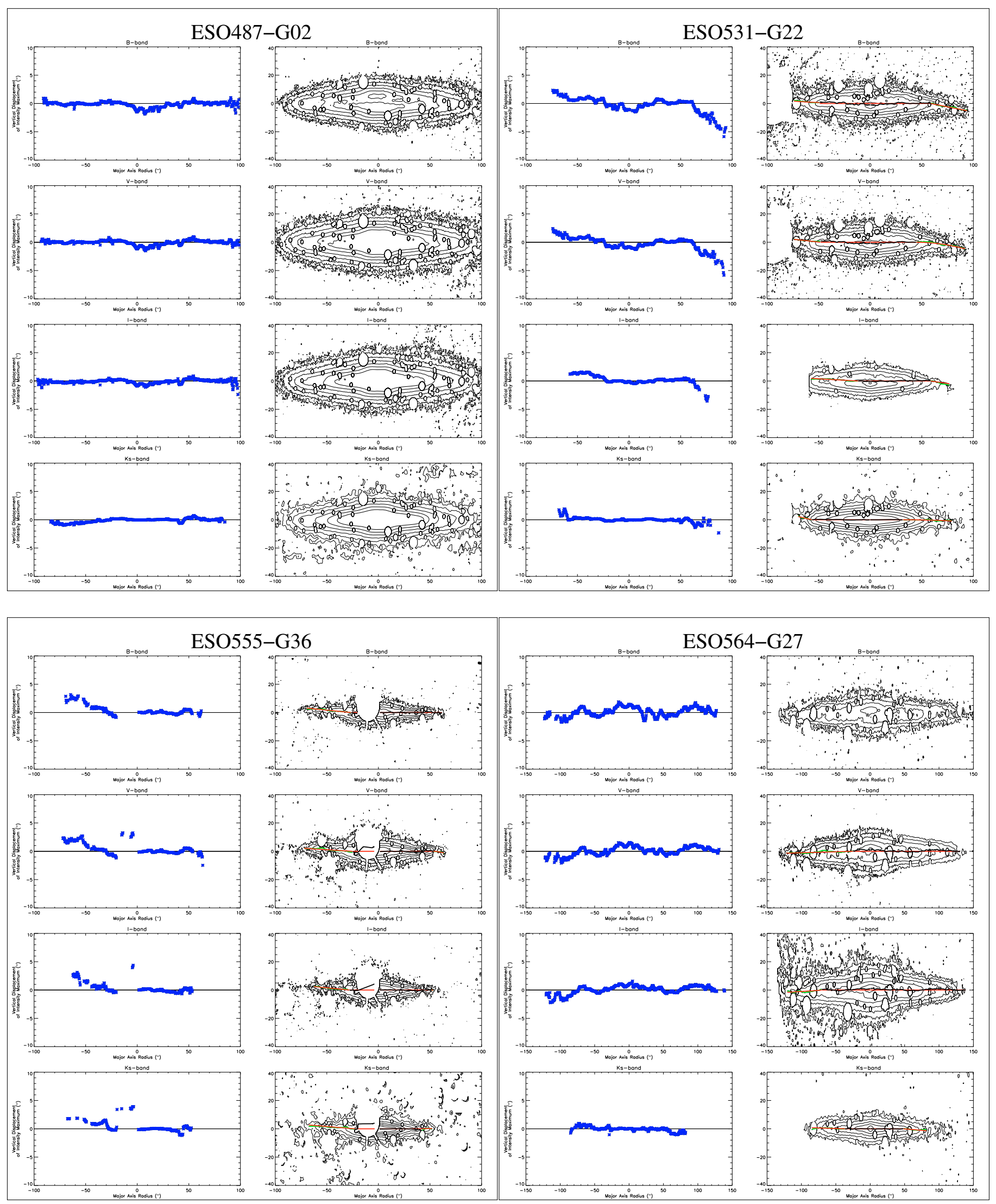

Fig. 3. continued.

both wavelengths the frequency of warps is the same, i.e., close to $100 \%$ (Sancisi 1976; García-Ruiz 2001; García-Ruiz et al. 2002b; van der Kruit 2007).
As expected, the $\mathrm{N}$ - and S-like warp frequencies are similar, since these characteristics depend on the observer rather than the galaxy. We observe six galaxies with $\mathrm{N}$-like and four galaxies 
A. Guijarro et al.: Near-infrared and optical observations of galactic warps

Table 2. Warp parameters for our four filters $\left(\times 10^{4}\right)$ and degree of asymmetry for each warped galaxy, with warp values listed in boldface.

\begin{tabular}{|c|c|c|c|c|c|c|c|c|c|c|c|c|c|c|c|c|}
\hline \multirow[t]{2}{*}{ Galaxy } & \multicolumn{4}{|c|}{$B$ filter } & \multicolumn{4}{|c|}{$V$ filter } & \multicolumn{4}{|c|}{$I$ filter } & \multicolumn{4}{|c|}{$K_{\mathrm{s}}$ filter } \\
\hline & $\omega$ & $\omega_{\mathrm{R}}$ & $\omega_{\mathrm{L}}$ & $\alpha_{\mathrm{s}}$ & $\omega$ & $\omega_{\mathrm{R}}$ & $\omega_{\mathrm{L}}$ & $\alpha_{\mathrm{s}}$ & $\omega$ & $\omega_{\mathrm{R}}$ & $\omega_{\mathrm{L}}$ & $\alpha_{\mathrm{s}}$ & $\omega$ & $\omega_{\mathrm{R}}$ & $\omega_{\mathrm{L}}$ & $\alpha_{\mathrm{s}}$ \\
\hline ESO026-G06 & $1 \pm 3$ & $1 \pm 3$ & $-0 \pm 3$ & & $0 \pm 2$ & $1 \pm 2$ & $-1 \pm 2$ & & $2 \pm 3$ & $2 \pm 3$ & $1 \pm 3$ & & $-3 \pm 3$ & $-2 \pm 3$ & $-1 \pm 3$ & \\
\hline ESO0? & $-1 \pm 4$ & $0 \pm 4$ & $-1 \pm 4$ & & $-3 \pm 6$ & $-1 \pm 6$ & $-2 \pm 6$ & & $-1 \pm 4$ & $-1 \pm 4$ & $0 \pm 4$ & & $3 \pm 4$ & $1 \pm 4$ & $1 \pm 4$ & \\
\hline ESO1 & $3 \pm 2$ & $4 \pm 2$ & $-1 \pm 2$ & 1.0 & $3 \pm 2$ & $4 \pm 2$ & $0 \pm 2$ & 1.0 & $4 \pm 2$ & $4 \pm 2$ & $0 \pm 2$ & 1.0 & $2 \pm 2$ & $1 \pm 2$ & $0 \pm 2$ & - \\
\hline G18 & $-4 \pm 3$ & $-2 \pm 3$ & $-1 \pm 3$ & - & $-4 \pm 2$ & $-3 \pm 2$ & $-1 \pm 2$ & -1.0 & $0 \pm 3$ & $-1 \pm 3$ & $1 \pm 3$ & - & $1 \pm 3$ & $1 \pm 3$ & $0 \pm 3$ & - \\
\hline 322 & $4 \pm 2$ & $3 \pm 2$ & $1 \pm 2$ & 1.0 & $2 \pm 2$ & $3 \pm 2$ & $-1 \pm 2$ & 1.0 & $2 \pm 2$ & $3 \pm 2$ & $-1 \pm 2$ & 1.0 & $2 \pm 3$ & $2 \pm 3$ & $1 \pm 3$ & - \\
\hline & $17 \pm 6$ & $12 \pm 6$ & $5 \pm 6$ & 1.0 & $16 \pm 3$ & $12 \pm 3$ & $5 \pm 3$ & 0.4 & $11 \pm 6$ & $9 \pm 6$ & $3 \pm 6$ & 1.0 & $4 \pm 4$ & $4 \pm 4$ & $2 \pm 4$ & - \\
\hline & $29 \pm 6$ & $10 \pm 6$ & $19 \pm 6$ & 0.3 & $39 \pm 7$ & $16 \pm 7$ & $22 \pm 7$ & 0.2 & $28 \pm 3$ & $11 \pm 3$ & $17 \pm 3$ & 0.2 & $25 \pm 2$ & $10 \pm 2$ & $15 \pm 2$ & 0.2 \\
\hline & $-4 \pm 3$ & $-4 \pm 3$ & $0 \pm 3$ & -1.0 & & $-3 \pm 1$ & $1 \pm 1$ & -1.0 & & $-1 \pm 2$ & $0=$ & - & $0 \pm 2$ & $0 \pm 2$ & $0 \pm 2$ & - \\
\hline & $-6 \pm 5$ & $1 \pm 5$ & $-8 \pm 5$ & -1.0 & $-7 \pm 2$ & $-2 \pm 2$ & $-5 \pm 2$ & -0.4 & $-6 \pm 3$ & $-1 \pm 3$ & $-5 \pm 3$ & -1.0 & $-5 \pm 1$ & $-3 \pm 1$ & $-2 \pm 1$ & -0.2 \\
\hline & $5 \pm 1$ & $3 \pm 1$ & $1=$ & 0.5 & $5 \pm 1$ & $3 \pm 1$ & $2 \pm 1$ & 0.2 & $5 \pm 1$ & $3 \pm 1$ & $2 \pm$ & 0.2 & $4 \pm 1$ & $2 \pm 1$ & $1 \pm 1$ & 0.3 \\
\hline & $-1 \pm 1$ & $1 \pm 1$ & $-2 \pm 1$ & -1.0 & $-1 \pm 1$ & $1 \pm 1$ & $-2 \pm 1$ & -1.0 & $-1 \pm 2$ & $1 \pm 2$ & $-2 \pm 2$ & - & $-1 \pm 1$ & $-1 \pm 1$ & $-1 \pm 1$ & 0.0 \\
\hline & $-2 \pm 7$ & $-6 \pm 7$ & $4 \pm 7$ & & $4 \pm 5$ & $-1 \pm 5$ & $5 \pm 5$ & & $1 \pm 8$ & $-6 \pm 8$ & $7 \pm 8$ & & $9 \pm 9$ & $2 \pm 9$ & $8 \pm 9$ & \\
\hline & $-1 \pm 1$ & $-1 \pm 1$ & $-1 \pm 1$ & & $0 \pm 1$ & $0 \pm 1$ & $0 \pm 1$ & & $0 \pm 0$ & $0 \pm 0$ & $0 \pm 0$ & & $2 \pm 2$ & $1 \pm 2$ & $2 \pm 2$ & \\
\hline & $0 \pm 1$ & $0 \pm 1$ & $0 \pm 1$ & & $1 \pm 1$ & $1 \pm 1$ & $1 \pm 1$ & & $-1 \pm 2$ & $0 \pm 2$ & $0 \pm 2$ & & $-2 \pm 2$ & $-1 \pm 2$ & $-1 \pm 2$ & \\
\hline $\mathrm{G} 25$ & $0 \pm 1$ & $2 \pm 1$ & $-1 \pm 1$ & 1.0 & $0 \pm 1$ & $2 \pm 1$ & $-1 \pm 1$ & 1.0 & $-2 \pm 2$ & $1 \pm 2$ & $-4 \pm 2$ & -1.0 & $-4 \pm 2$ & $-1 \pm 2$ & $-3 \pm 2$ & -1.0 \\
\hline G31 & $8 \pm 9$ & $1 \pm 9$ & $7 \pm 9$ & & $4 \pm 4$ & $2 \pm 4$ & $3 \pm 4$ & & $7 \pm 8$ & $1 \pm 8$ & $6 \pm 8$ & & $0 \pm 3$ & $-3 \pm 3$ & $3 \pm 3$ & \\
\hline G02 & $-1 \pm 3$ & $0 \pm 3$ & $0 \pm 3$ & & $-1 \pm 2$ & $0 \pm 2$ & $-1 \pm 2$ & & $1 \pm 2$ & $0 \pm 2$ & $1 \pm 2$ & & $4 \pm 4$ & $0 \pm 4$ & $4 \pm 4$ & \\
\hline $\mathrm{G} 22$ & $-20 \pm 6$ & $-14 \pm 6$ & $-6 \pm 6$ & -0.4 & $-16 \pm 4$ & $-11 \pm 4$ & $-5 \pm 4$ & -0.4 & $-11 \pm 4$ & $-4 \pm 4$ & $-7 \pm 4$ & -0.3 & $-6 \pm 2$ & $-2 \pm 2$ & $-4 \pm 2$ & -0.3 \\
\hline & $-10 \pm 4$ & $0 \pm 4$ & $-11 \pm 4$ & -1.0 & $-12 \pm 8$ & $-1 \pm 8$ & $-11 \pm 8$ & -1.0 & $-12 \pm 5$ & $-1 \pm 5$ & $-11 \pm 5$ & -1.0 & $-10 \pm 5$ & $-2 \pm 5$ & $-9 \pm 5$ & -1.0 \\
\hline $4-G 27$ & $3 \pm 3$ & $0 \pm 3$ & $2 \pm 3$ & - & $4 \pm 3$ & $2 \pm 3$ & $3 \pm 3$ & 1.0 & $3 \pm 2$ & $1 \pm 2$ & $2 \pm 2$ & 1.0 & $-5 \pm 1$ & $-3 \pm 1$ & $-2 \pm 1$ & -0.2 \\
\hline
\end{tabular}

Table 3. Warp parameters as a function of passband.

\begin{tabular}{|c|c|c|c|c|c|c|c|c|c|c|c|c|c|c|}
\hline \multirow[t]{2}{*}{ Galaxy name } & \multirow[b]{2}{*}{ Side } & \multirow[b]{2}{*}{$\begin{array}{c}r_{\text {last }} \pm 1\left({ }^{\prime \prime}\right) \\
(3)\end{array}$} & \multicolumn{4}{|c|}{$y( \pm 0.5)$} & \multicolumn{4}{|c|}{$A( \pm 1)$} & \multicolumn{4}{|c|}{$C( \pm 0.01)$} \\
\hline & & & $\begin{array}{c}B \\
(4)\end{array}$ & $\begin{array}{c}V \\
(5)\end{array}$ & $\begin{array}{c}I \\
(6)\end{array}$ & $\begin{array}{l}K_{\mathrm{s}} \\
(7)\end{array}$ & $\begin{array}{c}B \\
(8)\end{array}$ & $\begin{array}{l}V \\
(9)\end{array}$ & $\begin{array}{c}I \\
(10)\end{array}$ & $\begin{array}{c}K_{\mathrm{s}} \\
(11)\end{array}$ & $\begin{array}{c}B \\
(12)\end{array}$ & $\begin{array}{c}V \\
(13)\end{array}$ & $\begin{array}{c}I \\
(14)\end{array}$ & $\begin{array}{c}K_{\mathrm{s}} \\
(15)\end{array}$ \\
\hline \multirow[t]{2}{*}{ ESO142-G24 } & Right & 110 & -1 & -1.5 & 1 & 0 & 108 & 106 & 103 & - & 0.17 & 0.12 & 0.27 & - \\
\hline & Left & 103 & 0 & 0.5 & 0 & 0 & -105 & -103 & -104 & - & 0.10 & 0.04 & 0.08 & - \\
\hline \multirow[t]{2}{*}{ ESO157-G18 } & Right & 90 & -1.5 & -2 & -1 & 0 & 70 & 68 & - & - & -0.06 & -0.07 & - & - \\
\hline & Left & 92 & 0.5 & 0.5 & 0 & 1 & -81 & -115 & - & - & -0.05 & 0.04 & - & - \\
\hline \multirow[t]{2}{*}{ ESO201-G22 } & Right & 71 & 0 & 0.5 & 0.5 & 0 & 46 & 63 & 29 & - & 0.07 & 0.10 & - & - \\
\hline & Left & 72 & 1 & 1 & 0.5 & 0 & -66 & -86 & -63 & - & 0.05 & 0.21 & 0.02 & - \\
\hline \multirow{2}{*}{ ESO202-G35 } & Right & 82 & 2 & 2 & 2 & 0 & 30 & 33 & 43 & - & 0.90 & 0.07 & 0.05 & - \\
\hline & Left & 72 & -2 & -2 & -2 & -1.5 & -41 & -44 & -45 & - & 0.07 & 0.07 & 0.09 & - \\
\hline \multirow{2}{*}{ ESO235-G53 } & Right & 70 & 7 & 6 & 5 & 6 & 27 & 36 & 30 & 30 & 1.52 & 0.16 & 0.15 & 0.39 \\
\hline & Left & 70 & -7 & -5 & -6 & -4 & -11 & -35 & -35 & -39 & 0.11 & 0.16 & 0.11 & 0.10 \\
\hline \multirow[t]{2}{*}{ ESO240-G11 } & Right & 180 & -5.5 & -5.5 & -3 & 0 & 101 & 132 & - & - & - & - & - & - \\
\hline & Left & 165 & -0.5 & -1.5 & -1 & 0 & - & -103 & - & - & 0.00 & 0.02 & - & - \\
\hline \multirow[t]{2}{*}{ ESO288-G25 } & Right & 67 & -0.5 & -1 & -3.5 & -2.5 & 65 & 60 & 60 & 43 & -0.08 & -0.09 & -0.49 & -0.04 \\
\hline & Left & 73 & 2.5 & 2 & 1 & 0 & -26 & -28 & -29 & - & -0.03 & -0.05 & -0.02 & - \\
\hline \multirow[t]{2}{*}{ ESO311-G12 } & Right & 118 & 1.5 & 1 & 1 & 0 & 46 & 32 & 47 & 32 & 0.03 & 0.02 & 0.02 & 0.02 \\
\hline & Left & 102 & - & - & - & 0 & -49 & -75 & -63 & -60 & 0.01 & 0.06 & 0.03 & 0.02 \\
\hline \multirow[t]{2}{*}{ ESO340-G08 } & Right & 37 & 0 & 0 & 0 & 0 & 64 & 59 & - & - & -0.08 & -0.12 & - & - \\
\hline & Left & 39 & 0 & 0 & 0 & 0 & -50 & -50 & - & - & -0.05 & -0.05 & - & - \\
\hline \multirow[t]{2}{*}{ ESO416-G25 } & Right & 63 & 1.5 & 1.5 & 1.5 & -3 & 51 & 51 & 51 & 59 & 1.00 & 0.68 & 0.61 & -0.68 \\
\hline & Left & 69 & 0 & 0 & - & 1 & - & - & -46 & -54 & - & - & -0.04 & -0.06 \\
\hline \multirow[t]{2}{*}{ ESO531-G22 } & Right & 88 & -4 & -3.5 & - & -2.5 & 58 & 44 & 58 & 44 & -0.14 & -0.12 & -0.11 & - \\
\hline & Left & 69 & 2 & 1.5 & - & 2 & -39 & -48 & -12 & -57 & -0.05 & -0.04 & -0.04 & -0.14 \\
\hline \multirow[t]{2}{*}{ ESO555-G36 } & Right & 52 & 0 & 0 & 0 & 0 & - & 53 & 50 & - & - & - & -0.07 & - \\
\hline & Left & 69 & 2.5 & 2 & - & 1.5 & -28 & -25 & -28 & -21 & -0.04 & -0.02 & -0.04 & -0.02 \\
\hline \multirow[t]{2}{*}{ ESO564-G27 } & Right & 117 & 0 & 0.5 & 0.5 & -1 & - & - & - & 48 & - & - & -0.04 & -0.04 \\
\hline & Left & 83 & 0 & -1 & -0.5 & -0.5 & - & -25 & -70 & -37 & - & 0.01 & 0.02 & -0.02 \\
\hline
\end{tabular}

Notes. (3) Radius of the last measured point in $K_{\mathrm{s}}(\operatorname{arcsec})$; (4)-(7) $y$ at the same point (arcsec); (8)-(11) warp starting points (arcsec); (12)-(15) asymptotic slope.

with S-like warps. The frequency of asymmetric warps is lower: ESO416-G25 exhibits a U-like warp, while ESO240-G11 and ESO555-G36 show L-like warps. Therefore, the most frequent warp morphologies are N- and S-like warps (see also Ann \& Park 2006).

The distribution of warped galaxies as a function of morphological type is shown in Fig. 5, where the hatched area shows all galaxies and the cross-hatched area covers warped galaxies.
The warp frequency does not seem to depend significantly on galaxy morphology, except for S0 and very-late-type galaxies, which both yield lower frequencies.

A noticeable result from Sánchez-Saavedra et al. (2003) was that S0 galaxies are generally not warped. This places strong constraints on any hypothesis proposed to explain warps. Among our sample galaxies, ESO311-G12 has a small warp in both the optical and the NIR, but both of the other S0 sample galaxies, 


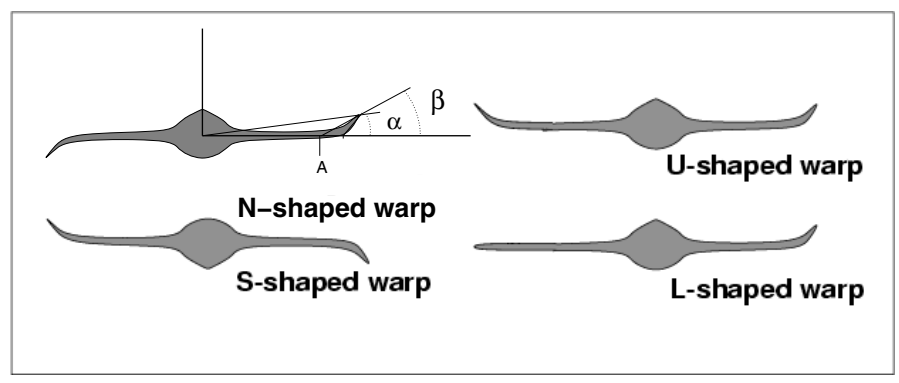

Fig. 4. Definitions of angles, parameters, and types of warps.

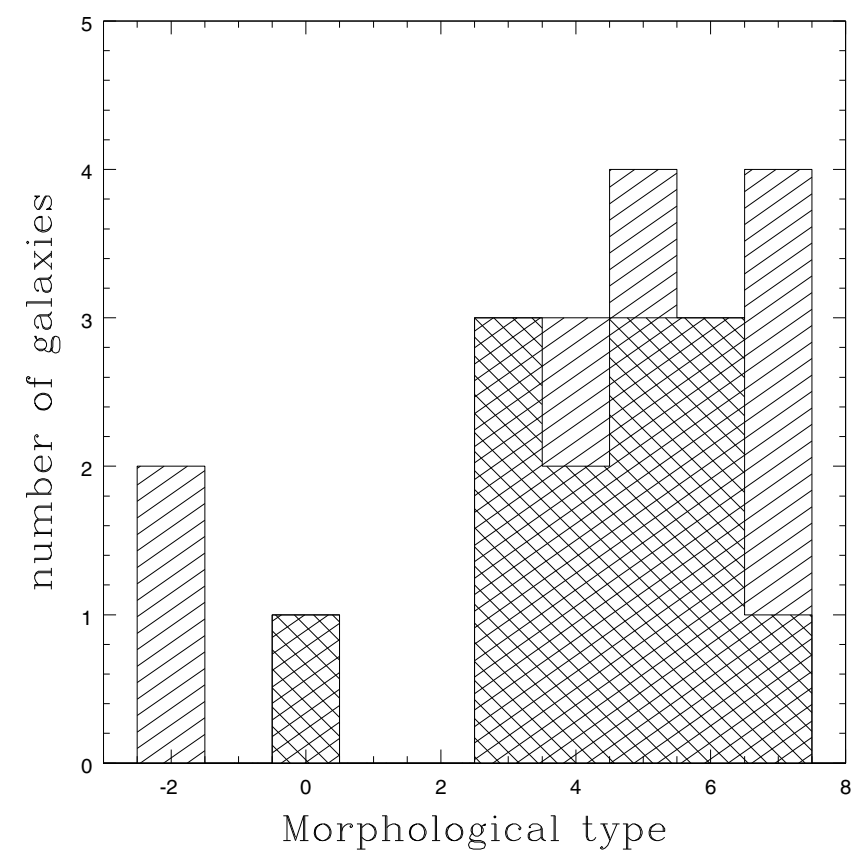

Fig. 5. Morphological-type distribution of our warped galaxies. Hatched area: all galaxies. Cross-hatched area: Warped galaxies.

ESO358-G26 and ESO358-G29, do not exhibit any detectable warps. Although our sample only contains three S0 galaxies, so that we cannot draw any statistical conclusions on that basis, our results are not in contradiction to previously published results.

Since we have data in both optical and NIR bands, we can compare both, enabling us to either detect or reject colour dependences in the warps. This comparison is difficult, however, because of inherent uncertainties and because the differences as a function of wavelength are not very large. Five of our galaxies (ESO235-G53, ESO288-G25, ESO311-G12, ESO416-G25, and ESO555-G36) show similar warp curves in all four observed bands, one galaxy (ESO531-G22) is warped in all four filters but exhibits a clearly weaker IR warp, one galaxy (ESO564-G27) is warped in the $V, I$, and $K_{\mathrm{S}}$ filters, and six galaxies (ESO142-G24, ESO157-G18, ESO201-G22, ESO202-G35, ESO240-G11, and ESO340-G08) are warped in the optical bands but not in the IR.

Since NIR profiles reach, in general, smaller radii than their optical counterparts, it is not straightforward to compare the warp distortion at different wavelengths. To do so, we compared the value of the fitted warp curve's $y$ at the same radial distance in each band, for which we adopted the last measured point in $K_{\text {s }}$ (i.e., the $h$ parameter of Ann \& Park 2006): see Table 3, Col.3. Based on this table, we appreciate that the optical $B$ and $V$ bands do not exhibit significant differences. However, we find a
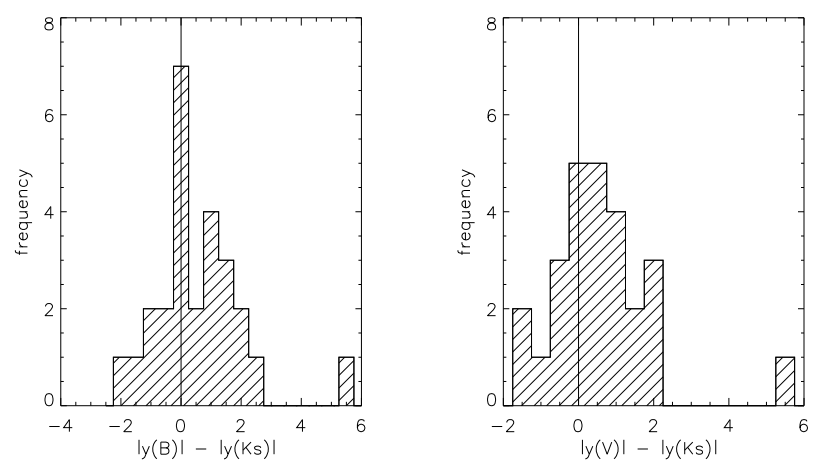

Fig. 6. Histogram of $y(B)-y\left(K_{\mathrm{s}}\right)$ and $y(V)-y\left(K_{\mathrm{s}}\right)$ at the last measured point in $K_{\mathrm{s}}$.

difference between $V$ and $K_{\mathrm{s}}: y(V)$ is usually greater than $y\left(K_{\mathrm{s}}\right)$ (in 14 of 22 cases; see Fig. 6). On the other hand, $y\left(K_{\mathrm{s}}\right)$ is greater than $y(V)$ for only six of the 22 cases. This difference can be quite large. The most extreme case is ESO240-G11 (right-hand side), where the $y(V)-z\left(K_{\mathrm{s}}\right)=5.5^{\prime \prime}$. We find a marginal tendency for NIR warps to have lower absolute values of the fit parameter $C$. Our attempts at statistically detecting a colour dependence of the observed warps are, therefore, inconclusive. A better understanding would require more studies of this type. If the colour dependence were eventually confirmed, mechanisms acting directly on the gas as the perturbing agent should be favoured. A colour difference has already been found by Florido et al. (1991). They also noticed that the dust lane in NGC 4013 is much more strongly warped than its optical disc. Recently, a study of 2MASS star counts in the Milky Way (Reyle et al. 2009) confirmed that the Galactic warp is less obvious in stars than in the gas and that the disc's shape is different at negative and positive longitudes. However, other authors have not been able to confirm such a difference (e.g., Momany et al. 2006, for the Milky Way).

Figure 7 shows the behaviour of the warp angle $\beta$ as a function of passband. This angle represents the strength of the warp, i.e. how much the outer disc deviates from the plane defined by the inner (unwarped) disc. Its highest value is obtained for galaxies with revised Hubble type $T=3$, corresponding to Sb. For these galaxy types, a tendency exists toward lower values of $\beta$ in the NIR filter than in the $B$ band. The starting point of the warp, $A$, does not appear to be a function of wavelength.

Kregel et al. (2002) analysed and used the same optical observations discussed here to study the flattening and truncation of the stellar discs. We take their measurements of the truncation radius (see van der Kruit 2007, for definition), $R_{\max }$, in the $I$ band for 11 of our warped galaxies. It would be very useful to compare this truncation radius with the starting radius of the warp $(A)$, because the relation between warps and disc truncations in edge-on galaxies (van der Kruit 2007), if any, is unclear. Obviously we obtain from this comparison that the warp radius, $A$, is approximately equal to $0.8 R_{\max }$. Van der Kruit (2007) concludes that when an HI warp is present, it starts at approximately $1.1 R_{\max }$. Therefore it appears that optical warps always start earlier than HI warps (HI warps start at approximately $1.4 \mathrm{~A}$ ), although we note that the low resolution of the HI data makes it difficult to detect low-amplitude warps.

Kregel et al. (2004) also presented HI synthesis observations of 15 edge-on galaxies. They have seven galaxies in common with our sample, of which ESO487-G02 was observed with insufficient sensitivity, and five galaxies are found to have a 

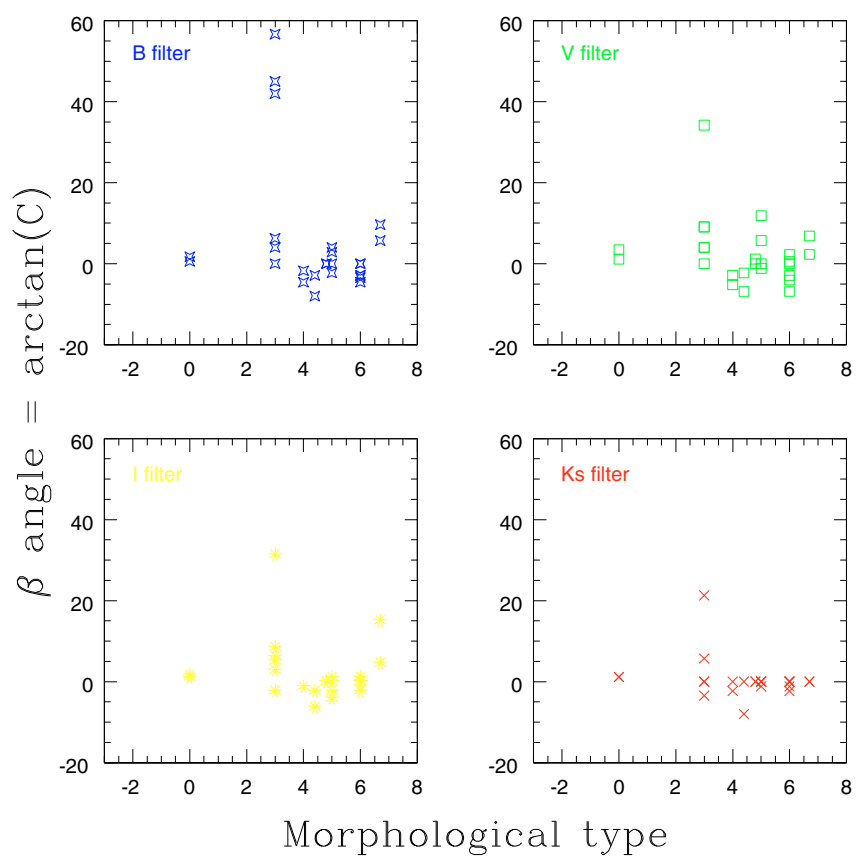

Fig. 7. Distribution of warp angle as a function of revised Hubble type.

warped HI disc. We now analyse the objects in common with Kregel et al. (2004) for which warps have been detected either by them, by us, or by both. They find that the neutral hydrogen distribution in ESO142-G24 appears warped on both sides of the galaxy, following a similar pattern to the stellar disc. In our optical observations, the warp starts at 106" in the south and 104" in the north, but on the basis of our NIR observations, we could not detect the same behaviour. Instead, we observe a corrugated disc.

Kregel et al. (2004) find that ESO157-G18 is lopsided, which may be related to the small companion, APMBGC157+052+052, although this galaxy had no previously determined redshift. They did not detect any clear HI warp. We also detected some differences between both sides of this galaxy. Our warp curves exhibit a similar behaviour in all optical bands, while in the NIR the warp is not clear.

ESO201-G22 shows a similar warp curve in HI and in all optical bands, but it is uncertain in the NIR, where the data are not as deep as in the optical. The $K_{\mathrm{s}}$-band warp curve finishes at a smaller radius than in the optical bands. The bright star on the eastern (left-hand) side of the galaxy hinders our analysis of this region.

ESO240-G11 is very interesting because a region in the galaxy's northwest (right) is clearly warped in the $B$ and $V$ bands, but the warp is not as marked in the NIR. Kregel et al. (2004) detected warping of the HI layer and they point out that the HI warp can be traced by faint emission in the I-band image. In the optical bands, the dust lane is not completely coincident with the galactic plane, which means that this galaxy is either not perfectly edge-on or that it has a warped or asymmetric bulge, which also results in a rather large error bar. On the southeastern (left-hand) side, the warp radius is $103 \pm 2^{\prime \prime}$ with $\beta=-2.3^{\circ}$, and on the northwestern side this radius is $132.3 \pm 0.6^{\prime \prime}$ and $\beta-63.7^{\circ}$, so this galaxy exhibits an L-like warp. The "elbow" where the warp begins in a given direction turns back to the mean plane and ends in the opposite hemisphere should show the beginning of the spiral arms. A more detailed study of corrugations in the galaxy's stellar and dust discs would also be very interesting.

The U-like type galaxy ESO416-G25 shows a similar warp curve in all bands, including in HI and the NIR.

The final galaxy also studied by Kregel et al. (2004) is ESO564-G27. This is a clearly corrugated Sc galaxy with some bright foreground stars projected onto the major axis, so it is very difficult to obtain robust conclusions about the stellar warp. Kregel et al. (2004) find that the neutral hydrogen is rather symmetric and extends further than the stellar disc. It is warped on both sides, with the warp apparently starting beyond the stellar disc. We cannot compare this with our results because of the large uncertainties.

Then we can conclude from this comparative study that 5 out of 6 galaxies have an HI warp (only ESO157-G18 does not show any clear HI warp), and these 5 galaxies have an optical warp, while in the NIR the presence of a warp is unclear or absent in all of them. This would indicate that the old star population is less warped than the gas and the more recently born stars.

We calculated $\alpha_{\mathrm{s}}$ - see Eq. (3) - for all warped galaxies, and the results are shown in Table 2 . The asymmetry in the warp angles seems related to the warp's intrinsic properties. García-Ruiz et al. (2002b) show that the largest asymmetries are observed in galaxies that display obvious tidal features. We can only show, based on our sample, that the three galaxies that exhibit asymmetries, ESO235-G53, ESO311-G12, and ESO531-G22, all show a large warp amplitude, which supports the positive correlation found by García-Ruiz et al. (2002b). However, none of the galaxies in our sample are involved in strong tidal interactions.

\section{Discussion and conclusions}

Are all warps produced by the same mechanism? For example, gravitationally driven warps should exhibit no differences between gaseous and stellar morphologies, as opposed to mechanisms acting on the gas (e.g., magnetically or accretion-driven warps). Are the warp curves the same for different colours; i.e., is there any difference in shape and magnitude at different wavelengths?

The present study suggests that, in some of the observed galaxies, the warp is smaller at NIR wavelengths than in the optical. This - combined with the important result of SánchezSaavedra et al. (2003) that lenticulars do not exhibit warps (which is also compatible with the results in this paper) - suggests that gas seems to be a necessary ingredient in the development of warps. We have not been able to robustly confirm the absence of warps in S0-type galaxies because our sample only contains three S0s. Therefore, we cannot draw any statistical conclusions on that basis, but our results (at least) do not contradict previously published results that most (if not all) lenticulars are not warped. This must be explained by some theoretical model. Models based on gravity alone might have significant difficulties to explain these observations. A recent study using 2MASS star counts in the Milky Way (Reylé et al. 2009) shows that the warp is less pronounced in stars than in the gas, and it also shows different disc shapes at negative and positive longitudes. The magnetic model of Battaner et al. (1990) is consistent with all of these observations.

Our results show that NIR warps exist with a frequency that is nearly the same as that of optical warps. This suggests that warps are long-lived structures that were formed in the early stages of the evolution of most galaxies. Since warps can be dissipated on a time scale close to the galaxy's rotation period, this indicates that the responsible mechanism, whatever it may 
be, seems to be acting permanently. The intergalactic magneticfield model remains an interesting possibility. It is well-known that a warp will disappear on a time scale of approximately 2 Gyr (Binney 1992). However, in the magnetic model of warps, the field configuration is not an initial condition but extragalactic fields act permanently. The extragalactic magnetic-field lines could be frozen in in the intergalactic medium, therefore corotating with galaxy clusters and presenting a relatively stable configuration of the field with respect to the warped galaxy.

The magnetic model was designed to explain $\mathrm{N}$ - and S-like warps ( $m=1$ modes), the most frequently occurring type $(51 \%$ out of $73 \%$ of warped discs in the large sample of 325 edge-on galaxies of Ann \& Park 2006). This model does not manage to explain $m=0$ warps (U-shaped profiles). They should be interpreted as coming from gradients in the extragalactic field, with characteristic lengths close to a galaxy's size. Generations of asymmetric warps through other mechanisms have been considered by Saha \& Jog (2006) and López-Corredoira et al. (2002). Other mechanisms have been proposed to induce warping. In fact, the variety of warp morphologies could come from different causes that may act differently from galaxy to galaxy. However, observations of NIR warps and direct comparison with their optical counterparts could provide some constraints.

The frequency of warped discs in our sample is very high $(65 \%)$. Given the difficulty of detecting warps with their lines of nodes in the plane of the sky, this suggests a fraction of nearly $100 \%$ for the frequency of warps in spirals. We have also been able to confirm the finding by Reshetnikov \& Combes (1999) that warps are equally present in all types of spirals, $T>0$.

The abrupt break between the undisturbed inner disc and the warped region (with a roughly constant slope) suggests that these two regions have different formation and evolutionary histories (e.g., van der Kruit 2007). However, van der Kruit concludes that HI warps start at $1.1 R_{\max }$. If this were the case, optical and NIR warps could not be observed. That they are indeed observed allows us to conclude that $R_{\max }>A$. Nevertheless, we cannot discard a possible relationship between truncations and warps. It appears that optical warps always start closer in than HI warps, although we note that the low resolution of the HI data makes it difficult to detect low-amplitude warps.

Acknowledgements. We are grateful for the helpful cooperation of the Ursuline Mothers during the period of this work. This paper has been supported by the "Plan Andaluz de Investigación" (FQM-108) and by the "Secretaría de Estado de Política Científica y Tecnológica" (AYA2000-1574).

\section{References}

Ann, H. B., \& Park, J.-C. 2006, NewA, 11, 293

Battaner, E. 1996, Astrophysical Fluid Dynamics (Cambridge University Press) Battaner, E., Florido, E., \& Sánchez-Saavedra, M. L. 1990, A\&A, 236, 1

Battaner, E., Garrido, J. L., Sánchez-Saavedra, M. L., \& Florido, E. 1991, A\&A, 251,402

Battaner, E., Florido, E., Jiménez-Vicente, J., Porcel, C., \& Sánchez-Saavedra, M. L. 1997, The Impact of Large Scale Near-IR Sky Surveys, ed. F. Garzón et al. (Dordrecht: Kluwer Academic Publishers), Series Astrophysics and Space Science, 210, 49
Binney, J. 1991, Dynamics of Disk Galaxies, ed. B. Sundelius, Goteborg, Sweden, 297

Binney, J. 1992, ARA\&A, 30, 51

Bosma, A. 1978, Ph.D. Thesis, Rijksuniversiteit Groningen, Netherlands, http: //nedwww.ipac. caltech.edu/leve15/March05/Bosma/ frames.html

Bosma, A. 1981, AJ, 86, 1791

Briggs, F. H. 1990, ApJ, 352, 15

Brinks, E., \& Burton, W.B. 1984, A\&A, 141, 195

Burke, B. F. 1957, AJ, 62, 90

Burton, W. B. 1988, Galactic and Extragalactic Radio Astronomy, ed. G. L.

Verschuur, \& K. I. Kellerman (Berlin and New York: Springer-Verlag), 295

Carter, B. S., \& Meadows, V. S. 1995, MNRAS, 276, 734

Corbelli, E., Schneider, S. E., \& Salpeter, E. E. 1989, AJ, 97, 390

Dekel, A., \& Shlosman, I. 1983, in Internal Kinematic \& Dynamics of Galaxies, ed. E. Athanassoula (Dordrecht: Reidel), IAU SYMP., 100, 187

de Grijs, R. 1997, Ph.D. Thesis, Rijksuniversiteit Groningen, Netherlands, http://dissertations.ub.rug.nl/faculties/science/1997/r. de.grijs/

de Grijs, R. 1998, MNRAS, 299, 595

Florido, E., Prieto, M., Battaner, E., Mediavilla, E., \& Sanchez-Saavedra, M. L. 1991, A\&A, 242, 301

García-Ruiz, I. 2001, Ph.D. Thesis, Rijksuniversiteit Groningen, Netherlands, http://dissertations.ub.rug.nl/faculties/science/2001/i. garcia-ruiz/

García-Ruiz, I., Kuijken, K., \& Dubinski, J. 2002a, MNRAS, 337, 459

García-Ruiz, I., Sancisi, R., \& Kuijken, K. 2002b, A\&A, 394, 769

Hunter, C., \& Toomre, A. 1969, ApJ, 155, 747

Jiménez-Vicente, J., Porcel, C., Sánchez-Saavedra, M. L., \& Battaner, E. 1997, Ap\&SS, 253, 225

Kahn, F. D., \& Woltjer, L. 1959, ApJ, 130, 705

Karachentseva, V. E., \& Karachentsev, I. D., 2000, Astron. Rep., 44, 501

Kerr, F. J. 1957, AJ, 62, 93

Kregel, M., van der Kruit, P. C., \& de Grijs, R. 2002, MNRAS, 334, 646

Kregel, M., van der Kruit, P. C., \& de Blok, W. J. G. 2004, MNRAS, 352, 768

Lauberts, A., \& Valentijn, E. A. 1989, The Surface Photometry Catalogue of the ESO-Uppsala Galaxies, ESO (ESO-LV)

López-Corredoira, M., Betancort-Rijo, J., \& Beckman, J. E. 2002, A\&A, 386, 169

López-Corredoira, M., Florido, E., Betancort-Rijo, J., et al. 2008, A\&A, 488, 511

Masset, F., \& Tagger, M. 1997, A\&A, 318, 747

Mathewson, D. S., Ford, V. L., \& Buchhorn, M. 1992, ApJS, 81, 413

Momany, Y., Zaggia, S., Gilmore, G., et al. 2006, A\&A, 451, 515

Porcel, C., Battaner, E., \& Jiménez-Vicente, J. 1997, A\&A, 322, 103

Reshetnikov, V., \& Combes, F. 1998, A\&A, 337, 9

Reshetnikov, V., \& Combes, F. 1999, A\&AS, 138, 101

Reshetnikov, V., Battaner, E., Combes, F., \& Jiménez-Vicente, J. 2002, A\&A, 382,513

Reylé, C., Marshall, D. J., Robin, A. C., \& Schultheis, M. 2009, A\&A, 495, 819

Rogstad, D. H., Lockhart, I. A., \& Wright, M. C. H. 1974, ApJ, 193, 309

Saha, K., \& Jog, C. J. 2006, A\&A, 446, 897

Sánchez-Saavedra, M. L., Battaner, E., \& Florido, E. 1990, MNRAS, 246, 458

Sánchez-Saavedra, M. L., Battaner, E., Guijarro, A., López-Corredoira, M., \& Castro-Rodríguez, N. 2003, A\&A, 399, 457

Sánchez-Salcedo, F. J. 2006, MNRAS, 365, 555

Sancisi, R. 1976, A\&A, 53, 159

Sparke, L. S. 1984, ApJ, 280, 117

Sparke, L. S., \& Casertano, S. 1988, MNRAS, 234, 873

Thuan, T. X., \& Gunn, J. E. 1979, PASP, 88, 543

Tubbs, A. D., \& Sanders, R. H. 1979, ApJ, 230, 736

van der Kruit, P. C. 2007, A\&A, 466, 883

Wainscoat, R. J., \& Cowie, L. L. 1992, AJ, 103, 33

Weinberg, M. D. 1998, MNRAS, 299, 499

Weinberg, M. D., \& Blitz, L. 2006, ApJ, 641, L33 